\title{
Elimination of the Actin-Binding Domain in Kelch-Like 1 Protein Induces T-Type Calcium Channel Modulation Only in the Presence of Action Potential Waveforms
}

\author{
Kelly A. Aromolaran, ${ }^{1,2}$ Kelly A. Benzow, ${ }^{3}$ Leanne L. Cribbs, ${ }^{4}$ \\ Michael D. Koob, ${ }^{3}$ and Erika S. Piedras-Rentería ${ }^{5,6}$ \\ ${ }^{1}$ Neuroscience Graduate Program, Loyola University Chicago, Chicago, IL 60153, USA \\ ${ }^{2}$ Department of Neuroscience, Kennedy Center, Albert Einstein College of Medicine, Room 610, 1300 Morris Park Avenue, \\ NY 10461, USA \\ ${ }^{3}$ Institute of Human Genetics, University of Minnesota, MMC 206, 420 Delaware Street SE, Minneapolis, MN 55455, USA \\ ${ }^{4}$ Office of Research Services, Room 4601, Building 102, 2160 S. First Avenue, Maywood, IL 60153, USA \\ ${ }^{5}$ Cellular and Molecular Physiology Department, Stritch School of Medicine, Loyola University Chicago, Room 4669, Building 102, \\ 2160 S. First Avenue, Maywood, IL 60153, USA \\ ${ }^{6}$ Neuroscience Institute, Stritch School of Medicine, Loyola University Chicago, Room 4669, Building 102, 2160 S. First Avenue, \\ Maywood, IL 60153, USA
}

Correspondence should be addressed to Erika S. Piedras-Rentería, erikapiedras@me.com

Received 6 April 2012; Accepted 30 May 2012

Academic Editor: Jesus Garcia

Copyright ( $) 2012$ Kelly A. Aromolaran et al. This is an open access article distributed under the Creative Commons Attribution License, which permits unrestricted use, distribution, and reproduction in any medium, provided the original work is properly cited.

\begin{abstract}
The Kelch-like 1 protein (KLHL1) is a neuronal actin-binding protein that modulates calcium channel function. It increases the current density of $\mathrm{Ca}_{\mathrm{v}} 3.2\left(\alpha_{1 \mathrm{H}}\right)$ calcium channels via direct interaction with $\alpha_{1 \mathrm{H}}$ and actin-F, resulting in biophysical changes in $\mathrm{Ca}_{\mathrm{v}} 3.2$ currents and an increase in recycling endosomal activity with subsequent increased $\alpha_{1 \mathrm{H}}$ channel number at the plasma membrane. Interestingly, removal of the actin-binding Kelch motif $\left(\Delta\right.$ Kelch) prevents the increase in $\mathrm{Ca}_{\mathrm{v}} 3.2$ current density seen with wild-type KLHL1 when tested with normal square pulse protocols but does not preclude the effect when tested using action potential waveforms (AP). Here, we dissected the kinetic properties of the AP waveform that confer the mutant Kelch the ability to interact with $\mathrm{Ca}_{\mathrm{v}} 3.2$ and induce an increase in calcium influx. We modified the action potential waveform by altering the slopes of repolarization and/or recovery from hyperpolarization or by changing the duration of the depolarization plateau or the hyperpolarization phase and tested the modulation of $\mathrm{Ca}_{\mathrm{v}} 3.2$ by the mutant $\Delta$ Kelch. Our results show that the recovery phase from hyperpolarization phase determines the conformational changes that allow the $\alpha_{1 \mathrm{H}}$ subunit to properly interact with mutant KLHL1 lacking its actin-binding Kelch domains, leading to increased Ca influx.
\end{abstract}

\section{Introduction}

Neuronal voltage-gated calcium channel function is central in processes such as neurotransmission and excitability, burst firing, intracellular signaling, and gene expression; thus, modulation of their activity can be physiologically relevant. The modulation of calcium channels by the actin cytoskeleton or actin-binding proteins $(\mathrm{ABP})$ is not well understood. However, recently, we reported a novel mechanism of modulation of $\mathrm{Ca}_{\mathrm{v}} 3.2$ T-type channel $\alpha_{1 \mathrm{H}}$ subunit by the actin-binding protein Kelch-like 1 (KLHL1) [1].

KLHL1 is a neuronal ABP member of the Kelch superfamily that contains their two signature motifs: a BTB/POZ domain involved in protein-protein interactions and a Kelch $\beta$-propeller region involved in actin binding $[2,3]$. This $\mathrm{ABP}$ is constitutively present in the nervous system, and it is involved in the modulation of neuronal structure and function; its genetic elimination results in loss of postsynaptic 


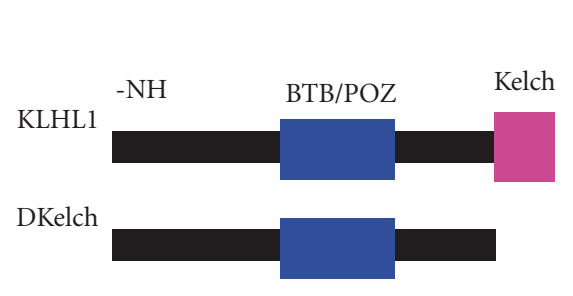

(a)

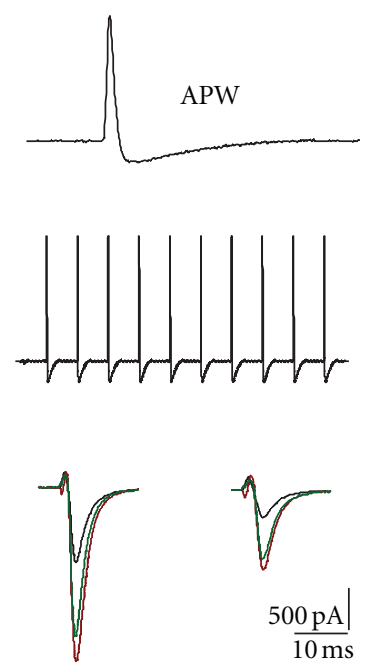

(c)

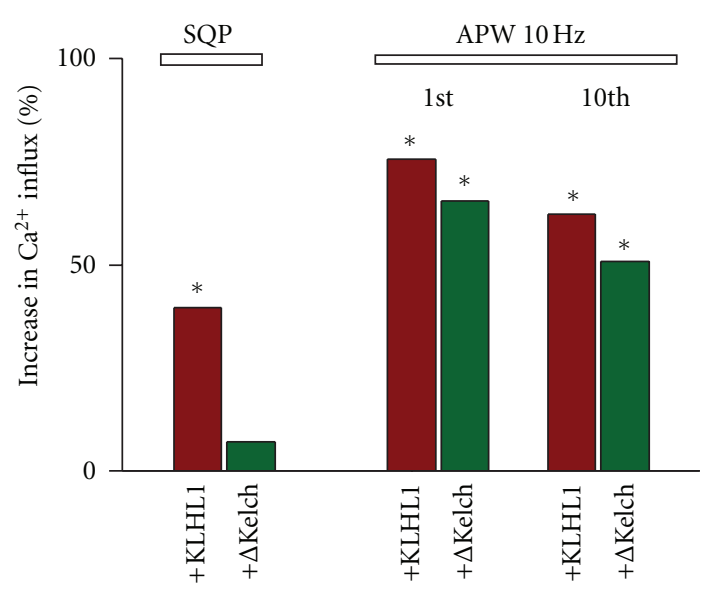

(d)

FIGURE 1: The wild-type KLHL1 actin-binding protein and the truncated mutant $\Delta$ Kelch both modulate calcium influx through Ca 3.2 upon stimulation with action potentials. (a) Cartoon illustrating the overall structure of KLHL1 and $\triangle$ Kelch; the amino terminus region (-NH), $\mathrm{BTB} / \mathrm{POZ}$, and Kelch domains are represented. (b) Example currents elicited by $\mathrm{Ca}_{\mathrm{v}} 3.2$ with a square pulse (SQP) from -100 to $-25 \mathrm{mV}$ under control conditions (black trace), or in the presence of KLHL1 (red trace) or $\triangle$ Kelch (green trace). (c) Single-action potential (AP) or $10 \mathrm{~Hz}$ action potential waveform (APW) protocols used throughout this work. Examples of the current traces elicited for each condition are shown below, at the 1st and 10th spike during the APW train. (d) Percentage of increase in calcium influx with respect to control.

structures, a progressive loss of motor coordination and gait abnormalities in mice [4].

We have reported that KLHL1 is a modulator of voltagegated calcium channels; it upregulates calcium current density of high- and low-voltage-gated channels $[1,5,6]$. We found that KLHL1 augmented the current density of $\mathrm{Ca}_{\mathrm{v}} 3.2$ (35\% increase) but not $\mathrm{Ca}_{\mathrm{v}} 3.1$ [1]. Indeed, we demonstrated in biochemistry assays that KLHL1 co-precipitates with $\alpha_{1 \mathrm{H}}$ in membrane fractions obtained from the HEK 293 overexpression system and from mouse brains [1], corroborating that the ABP and $\alpha_{1 \mathrm{H}}$ directly interact. We found that this modulation is done by a novel mechanism that involves increasing the number of functional channels at the plasma membrane and by a discrete alteration of the kinetics of $\tau_{\text {deactivation, }}$, resulting in increased calcium current density and calcium influx. The former effect occursvia enhanced channel reinsertion into the plasma membrane through the recycling endosome and requires the presence of polymerized actin [5].

In our examination of the function of the motifs in KLHL1, we tested a truncated mutant lacking the Kelch domains (located at the carboxyl terminus of the protein)
( $\Delta$ Kelch construct, Figure 1(a)). Interestingly, the elimination of this actin-binding motif in KLHL1 resulted in the elimination of mostly all of the upregulation of $\mathrm{Ca}_{\mathrm{v}} 3.2$ by the ABP. However, when calcium influx was elicited with a single-action potential (AP) or an action potential train waveform protocol (APW), the $\triangle$ Kelch mutant could still elicit the upregulatory effect [1]. Overall, these experiments indicated that the actin cytoskeleton is involved in the regulation of $\mathrm{Ca}_{\mathrm{v}} 3.2$ by KLHL1, as expected for an ABP, but they also suggested that additional regions in KLHL1 independent of the Kelch motif are involved in the direct interaction with the $\alpha_{1 \mathrm{H}}$ subunit. Here, we extend our studies to explore the specific properties of the action potential (AP) waveform that allow the interaction of the truncated KLHL1 mutant with $\mathrm{Ca}_{\mathrm{v}} 3.2$, resulting in increased calcium influx.

\section{Material and Methods}

2.1. Cell Culture and Transfection. Human embryonic kidney 293 cells stably transfected with the $\mathrm{Ca}_{\mathrm{v}} 3.2 \alpha_{1 \mathrm{H}}$ subunit were grown to $60 \%$ confluence and transfected using the calcium phosphate method [7] or polyethyleneimine (PEI) [8]. Cells 
were transfected with $1 \mu \mathrm{g}$ of human EGFP-KLHL1 cDNA or $\Delta$ Kelch cDNA $[1,2]$; EGFP cDNA was used to maintain equal transfection concentrations in control experiments.

2.2. Electrophysiology. Currents were recorded at 1-3 days after transfection using whole-cell patch clamp at room temperature as previously described $[1,5]$. Currents were recorded using an Axopatch 200B amplifier (Axon instruments, Union City, CA), and data were acquired at $1 \mathrm{kHz}$ and digitized at $10 \mathrm{kHz}$ using the Digidata $1322 \mathrm{~A}$ analog-to-digital converter. Currents were recorded in an external solution containing (in $\mathrm{mM}$ ) $5 \mathrm{CaCl}_{2}, 140 \mathrm{TEACl}, 10$ HEPES, and 10 glucose ( $\mathrm{pH} 7.4,300 \mathrm{mOsm}$ ). Pipettes pulled from borosilicate glass (Warner Instruments Inc., Hamden, CT) were filled with intracellular solution containing (in $\mathrm{mM}$ ) 108 $\mathrm{CsMeSO}_{3}, 4 \mathrm{MgCl}_{2}, 1$ Cs-EGTA, 9 HEPES, 5 ATP-Mg, 1 GTP-Li, and 15 phosphocreatine-TRIS (pH 7.4, $280 \mathrm{mOsm}$ ). Cells with series resistance $\left(R_{s}\right)<10 \mathrm{M} \Omega$ were used; $R_{s}$ was compensated online $(>80 \%)$.

Currents were elicited using a square pulse protocols from a holding potential $\left(V_{h}\right)=-100 \mathrm{mV}$ and depolarized for $150 \mathrm{~ms}$ to test potentials $\left(V_{t}\right)=-70$ to $+70 \mathrm{mV}$, in $5 \mathrm{mV}$ increments. The action potential waveform (APW) consisted of a digitized action potential that had a resting potential of $-70 \mathrm{mV}$, an upstroke of $116 \mathrm{mV} / \mathrm{ms}$ to a peak voltage of $+50 \mathrm{mV}$, followed by a repolarizing downstroke at $-65 \mathrm{mV} / \mathrm{ms}$ to a hyperpolarizing potential of $-90 \mathrm{mV}$. The repolarization from afterhyperpolarization (AHP) slope was $0.78 \mathrm{mV} / \mathrm{ms}$ to resting conditions (Figure 2(a), left).

The square pulse action potential waveform (SQAP) tested (Figure 2(a), right) consisted of a $V_{h}=-70 \mathrm{mV}$, instantaneous depolarization $(120 \mathrm{mV} / \mathrm{ms})$ to $+50 \mathrm{mV}$ for $3 \mathrm{~ms}$, followed by instantaneous repolarization to $-90 \mathrm{mV}$ for $50 \mathrm{~ms}$. Subsequent changes to the SQAP were as follows:

(a) changes in the duration of the depolarization step to $+50 \mathrm{mV}(0.5 \mathrm{~ms}$, Figure 3(a), blue trace);

(b) changes in the duration of afterhyperpolarization to $-90 \mathrm{mV}$ (6 ms, Figure 3(a), orange trace);

(c) changes in the repolarization slope (from peak voltage, $+50 \mathrm{mV}$ ) from instantaneous to $70 \mathrm{mV} / \mathrm{ms}$ (blue trace in Figure $4(\mathrm{a})$ ) or to $35 \mathrm{mV} / \mathrm{ms}$ (orange trace in Figure 4(a));

(d) changes in the afterhyperpolarization slope (AHP slope) (from peak negative voltage $=-90 \mathrm{mV}$ ) to resting potential at $20,0.75$ or $0.38 \mathrm{mV} / \mathrm{ms}$ slopes (Figure 5(a), blue, black and orange trace resp.);

(e) changes in both the repolarization and AHP slopes, resulting in the traces depicted in Figure 6(a), with the three repolarization slopes described in (c) and a $0.75 \mathrm{mV} / \mathrm{ms}$ afterhyperpolarization slope in all traces (Figure 6(a), black, blue and orange trace resp.);

(f) "action potential-like" waveforms, with repolarization ramp at $62 \mathrm{mV} / \mathrm{ms}$ and AHP slope ramp at $0.75 \mathrm{mV} / \mathrm{ms}$ (Figure $7(\mathrm{a})$, gray trace); repolarization ramp at $62 \mathrm{mV} / \mathrm{ms}$ and AHP slope ramp
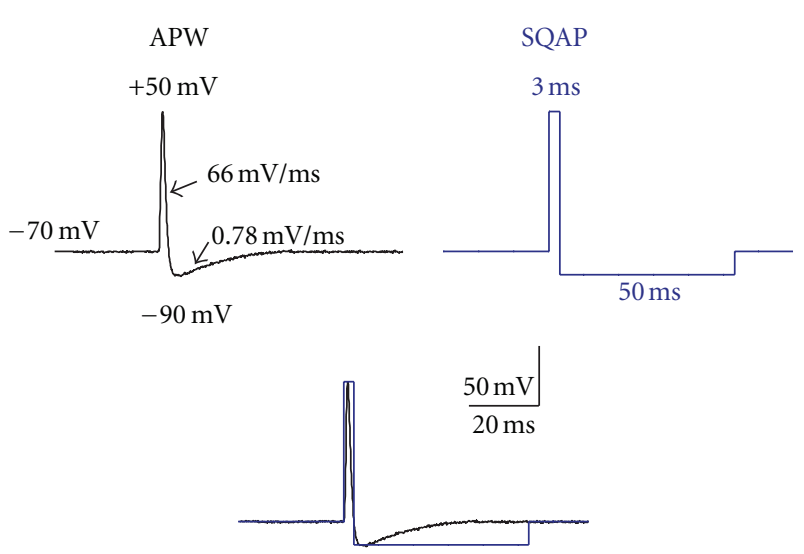

(a)

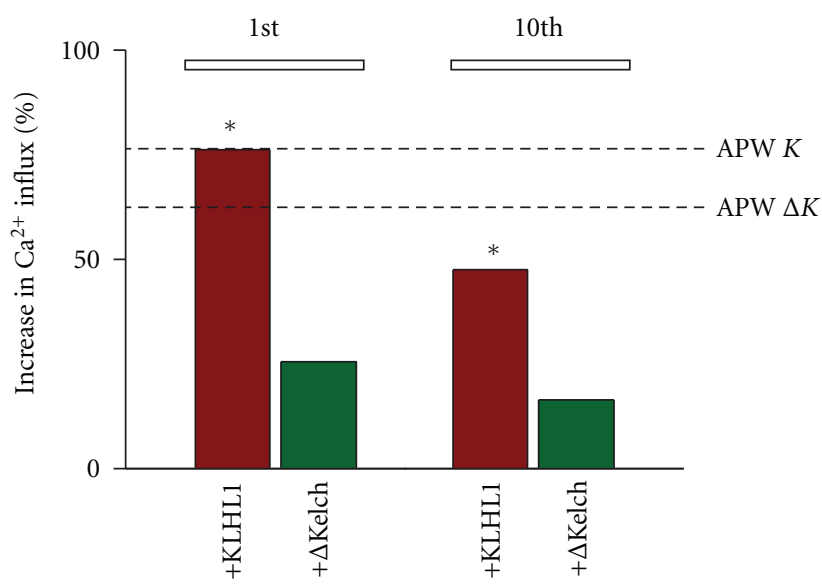

(b)

FIGURE 2: "Square" action potentials roughly similar to the original APW fail to enable upregulation of $\mathrm{Ca}_{\mathrm{v}} 3.2$ by $\Delta$ Kelch. (a) Original APW waveform was used (left) and square action potential was tested (SQAP). The overlapped signals is shown for comparison. (b) Percentage of increase in calcium influx with the SQAP with respect to control; the magnitude of the changes induced by the original APWs are shown with dotted lines $(K=$ KLHL1; $\Delta K=\Delta$ Kelch $)$; ${ }^{*} P<0.05$.

at $0.70 \mathrm{mV} / \mathrm{ms}$ (Figure $7(\mathrm{a})$, blue trace); repolarization ramp at $62 \mathrm{mV} / \mathrm{ms}$ and AHP slope ramp at $0.35 \mathrm{mV} / \mathrm{ms}$ (Figure $7(\mathrm{a})$, orange trace).

Total calcium influx was measured as the total charge elicited (in picocoulombs, pC) obtained by the integral of individual traces and normalized to cell size ( $\mathrm{pC} / \mathrm{pF})$. Data were analyzed with Clampfit 9 software (Axon Instruments). Results are presented as mean \pm SEM. Percentages of increase were calculated as the ratio of the experimental values $\left(\mathrm{Ca}_{\mathrm{v}} 3.2\right.$ in the presence of $\Delta$ Kelch or KLHL1) divided by the value of the $\mathrm{Ca}_{\mathrm{v}} 3.2$-mediated currents in the absence on KLHL1, expressed as percentage. Statistical significance was determined by $P<0.05$ using Student's $t$-test.

\section{Results}

3.1. Mutant KLHL1 Protein Lacking the Actin-Binding Domain (AKelch) Upregulates $\alpha_{1 H}$ T-Type Currents Only with Action Potential Waveforms. The elimination of this 


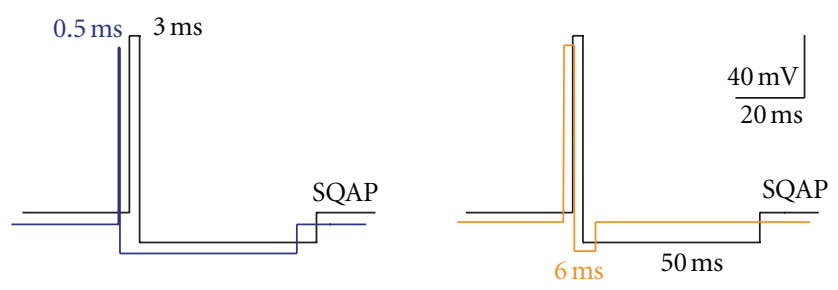

(a)

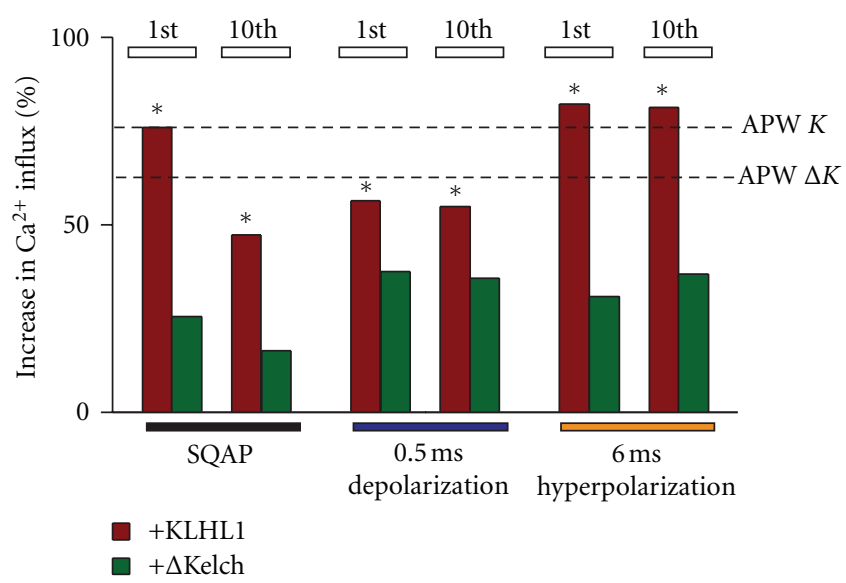

(b)

FIGURE 3: Changes in the duration of depolarization or hyperpolarization steps of the SQAP fail to enable upregulation of $\mathrm{Ca}_{\mathrm{v}} 3.2$ by $\Delta$ Kelch. (a) Square AP with a shorter depolarization phase $(0.5 \mathrm{~ms}$, blue trace) or hyperpolarization phase $(6 \mathrm{~ms}$, orange trace). (b) Percentage of increase in calcium influx with the three protocols, with respect to control (action potential waveform, APW; the magnitude of the changes induced by the original APWs is shown with dotted lines $(K=$ KLHL1; $\Delta K=\Delta$ Kelch $)) ;{ }^{*} P<0.05$.

actin-binding motif in KLHL1 results in the elimination of mostly all of the upregulation of $\mathrm{Ca}_{\mathrm{v}} 3.2$ by the $\mathrm{ABP}$, as described in [1] and summarized in Figure 1(b), where currents elicited with a square pulse protocol (SQP) from $-100 \mathrm{mV}$ to $-25 \mathrm{mV}$ resulted in upregulated currents in the presence of KLHL1 (red trace) compared to controls (black trace), in contrast to the mutant $\Delta$ Kelch (green trace). In contrast, when calcium influx was elicited with a singleaction potential (AP) or an action potential train waveform protocol (APW), the $\Delta$ Kelch mutant could still elicit the upregulatory effect (panel c). The data is summarized in panel (d), where it can be seen that the SQP protocol was overall less efficient at increasing calcium influx, and that an AP or APW protocol (in this case delivered at a frequency of $10 \mathrm{~Hz}$ ) could still elicit calcium influx increase in the presence of the $\Delta$ Kelch-truncated mutant. As expected, APWs delivered at $10 \mathrm{~Hz}$ or higher frequencies always elicited a concomitant current inactivation at the end of the train, as previously reported $[1,9]$. These experiments suggest that additional regions in KLHL1, independent of the Kelch motif, are involved in the direct interaction with $\alpha_{1 \mathrm{H}}$; therefore in the following sections, we dissect the properties

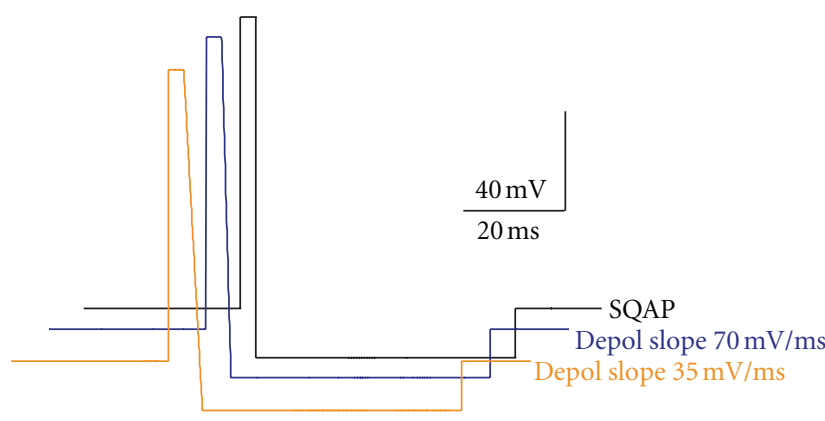

(a)

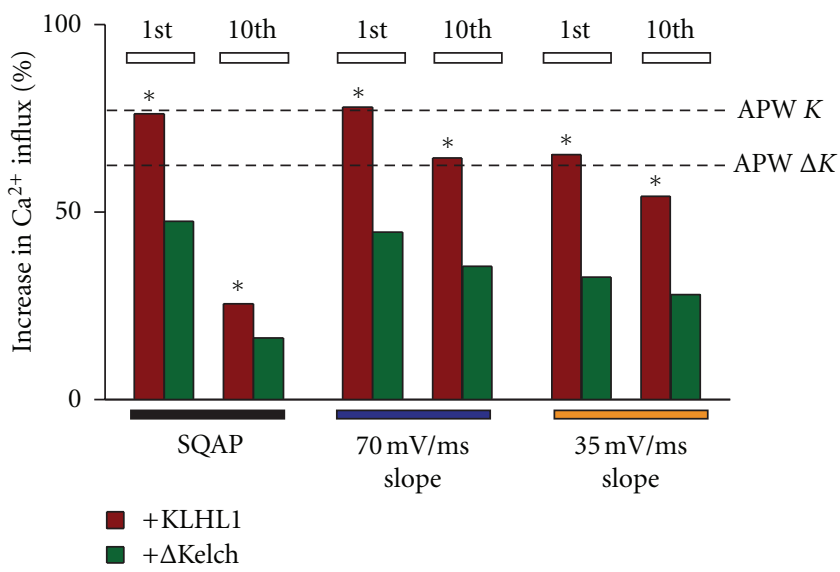

(b)

FIGURE 4: Changes in the repolarization slope of the SQAP fail to enable upregulation of $\mathrm{Ca}_{\mathrm{v}} 3.2$ by $\Delta$ Kelch. (a) SQAP waveforms with instantaneous repolarization (black), midrange depolarization slope $(70 \mathrm{mV} / \mathrm{ms}$, blue), and slower repolarization $(35 \mathrm{mV} / \mathrm{ms}$, orange). (b) Percentage of increase in calcium influx with the three waveforms with respect to control; the extent of the changes induced by the original APWs is shown with dotted lines $(K=$ KLHL1; $\Delta K=\Delta$ Kelch); ${ }^{*} P<0.05$.

of the action potential (AP) waveform that are important for the interaction of the mutant $\Delta$ Kelch with $\mathrm{Ca}_{\mathrm{v}} 3.2$.

\section{2. "Square" Action Potentials Roughly Similar to the Action} Potential Waveform (APW) Fail to Enable Upregulation of $\alpha_{1 H}$ by $\Delta$ Kelch. The upregulation of $\mathrm{Ca}_{\mathrm{v}} 3.2$ by KLHL1 or $\Delta$ Kelch can be observed when the stimulus protocol is an AP waveform, irrespective of the frequency of delivery. We explored the consequences of modifying the AP on the effect of KLHL1 and $\triangle$ Kelch on $\mathrm{Ca}_{\mathrm{v}} 3.2$-mediated calcium influx; APWs were delivered at $10 \mathrm{~Hz}$ throughout this study and are shown in all figures; however, only the values obtained for the first stimulus will be discussed for simplification purposes, as the effects are rather consistent at all frequencies throughout the study (up to $100 \mathrm{~Hz}$, not discussed here). The main effect of changes in the stimulation frequency is the presence of current inactivation proportional to the frequency used $[9,10]$.

The original AP waveform was obtained from a hippocampal neuron and digitized for its use as the waveform protocol (see Methods). To dissect the AP components that 


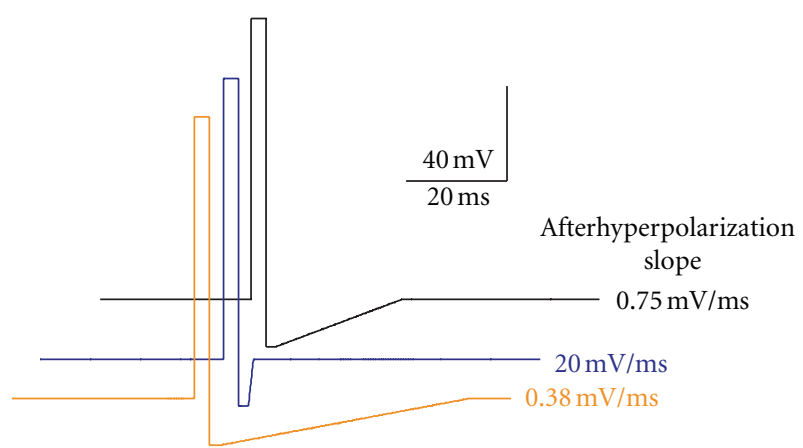

(a)

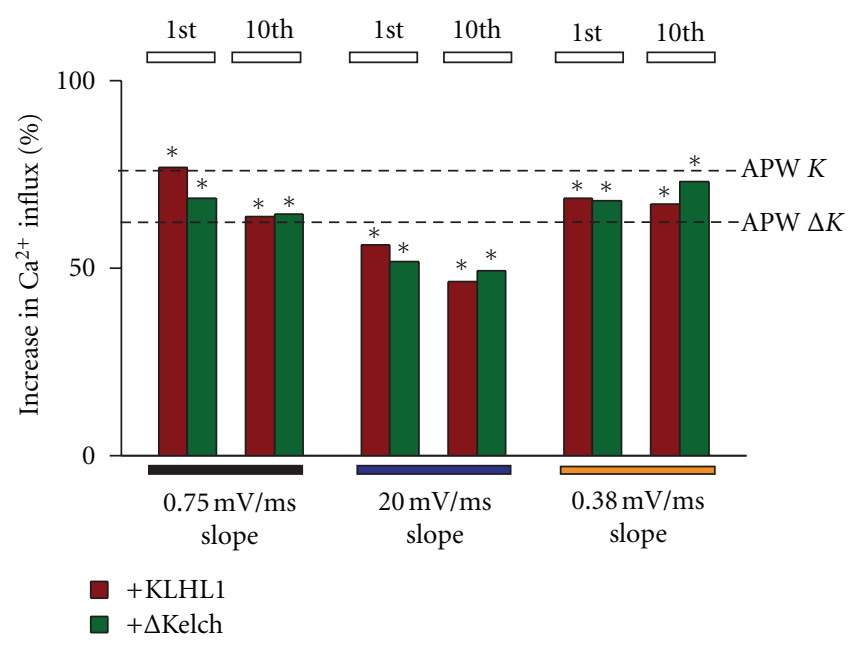

(b)

FIGURE 5: Changes in the afterhyperpolarization slope restore the $\Delta$ Kelch effect, increasing $\mathrm{Ca}^{2+}$ influx through $\mathrm{Ca}_{\mathrm{v}} 3.2$. (a) SQAP with afterhyperpolarization slopes of $20 \mathrm{mV} / \mathrm{ms}$ (blue), $0.75 \mathrm{mV} / \mathrm{ms}$ (black), and $0.35 \mathrm{mV} / \mathrm{ms}$ (orange). (b) Percentage of increase in calcium influx with the three waveforms with respect to control; the magnitude of the changes induced by the original APWs is shown with dotted lines $(K=\mathrm{KLHL} 1 ; \Delta K=\Delta$ Kelch $) ;{ }^{*} P<0.05$.

allow calcium influx increase in the presence of $\triangle$ Kelch, we first devised a rough "square action potential" or SQAP as depicted to the right in Figure 2(a), from the same $V_{h}$ as the AP waveform (APW) shown to the left, with an instantaneous upstroke to $+50 \mathrm{mV}$ followed by instantaneous repolarization back to $-90 \mathrm{mV}$ for $50 \mathrm{~ms}$. Both stimuli are shown overlapped in Figure 2(a) (bottom) for comparison.

Delivery of an SQAP resulted in the expected increase in calcium influx by KLHL1 ( $76 \%$ increase (first stimulus), $n=19, P<0.05)$, whereas $\Delta$ Kelch only elicited a $25 \%$ increase, not statistically significant compared to controls $(n=38)$. The values reached with a conventional APW are denoted in the figure by the dotted lines for each KLHL1 and $\Delta$ Kelch. Interestingly, $\Delta$ Kelch not only failed to elicit a significant calcium influx increase with the SQAP stimulus, but the extent of the increase was much smaller than that obtained with a regular APW; this effect could also be observed in the 10th stimulus with KLHL1 (shown in Figure $1(\mathrm{~d})$ ). We then proceeded to modify the duration

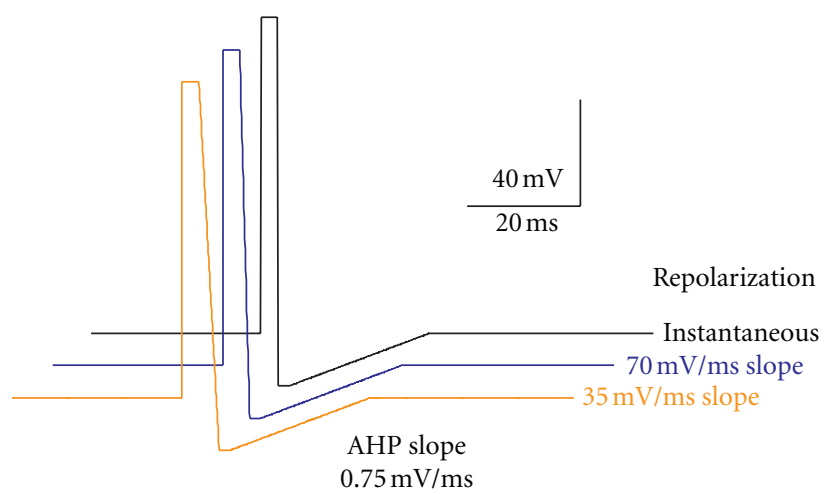

(a)

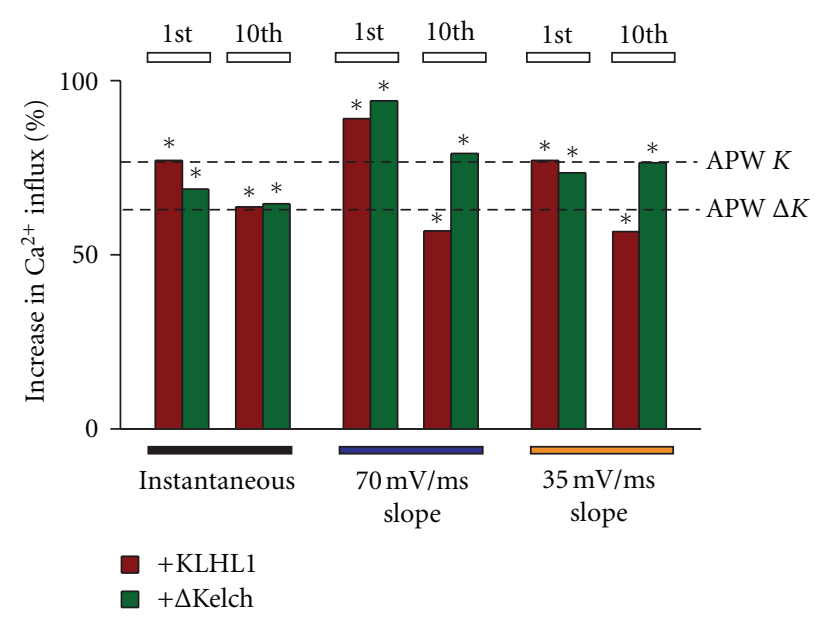

(b)

FIGURE 6: Changes in the repolarization slope in conjunction with an afterhyperpolarization slope synergize to enhance $\mathrm{Ca}^{2+}$ influx by $\Delta$ Kelch. (a) Protocols with a sloping afterhyperpolarization ramp of $0.75 \mathrm{mV} / \mathrm{ms}$ were combined with repolarizing slopes of 70 or $35 \mathrm{mV} / \mathrm{ms}$ (blue and orange, resp.), and compared with a waveform with instantaneous repolarization (black). (b) Percentage of increase in calcium influx with the three waveforms with respect to control; the magnitude of the changes induced by the original APWs is shown with dotted lines $(K=\mathrm{KLHL} 1 ; \Delta K=\Delta$ Kelch $) ;{ }^{*} P<0.05$.

of depolarization of the waveform (at $+50 \mathrm{mV}$ ) and the duration of the hyperpolarization at $-90 \mathrm{mV}$, as seen in panel (a) from Figure 3.

3.3. SQAPs with Shorter Duration at Depolarized Potentials or Shorter Times of Hyperpolarization Fail to Enable Upregulation of $\mathrm{Ca}_{v} 3.2$ by $\Delta$ Kelch. The SQAP was modified by decreasing the time spent at $+50 \mathrm{mV}$ from $3 \mathrm{~ms}$ to $0.5 \mathrm{~ms}$, as shown in Figure 3(a), left. The time spent at hyperpolarized potentials was also modified in a different protocol, which has reduced hyperpolarized potential duration (to $-90 \mathrm{mV}$ ), from $50 \mathrm{~ms}$ to $6 \mathrm{~ms}$ (Figure 3(a), right).

As observed in panel (b), none of the manipulations rescued the effect of $\Delta$ Kelch on $\mathrm{Ca}_{\mathrm{v}} 3.2$ as seen in figure (green bars). SQAP protocols elicited $25 \%$ of the influx of calcium by $\mathrm{Ca}_{\mathrm{v}} 3.2$ in the presence of $\Delta \operatorname{Kelch}(n=38)$, and decreasing the depolarization time increased influx to $38 \%$ 

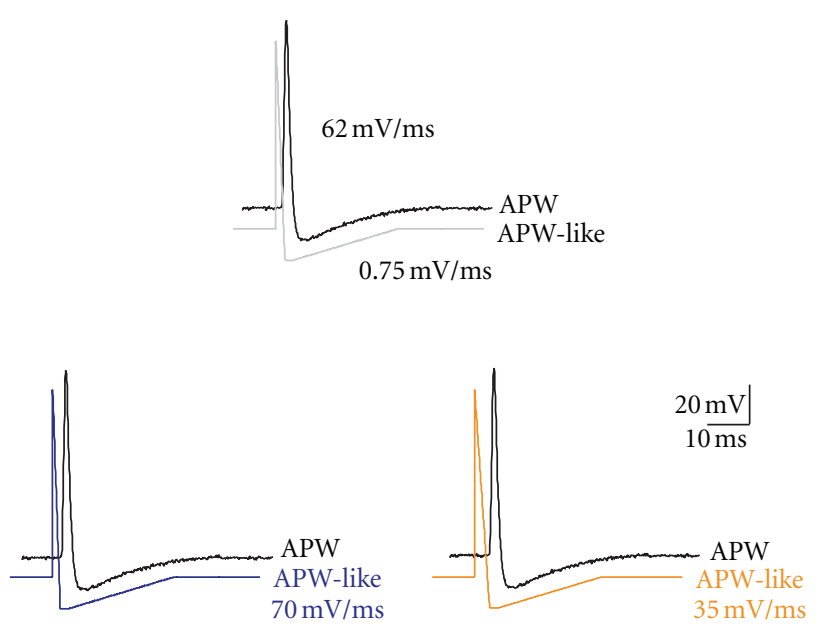

(a)

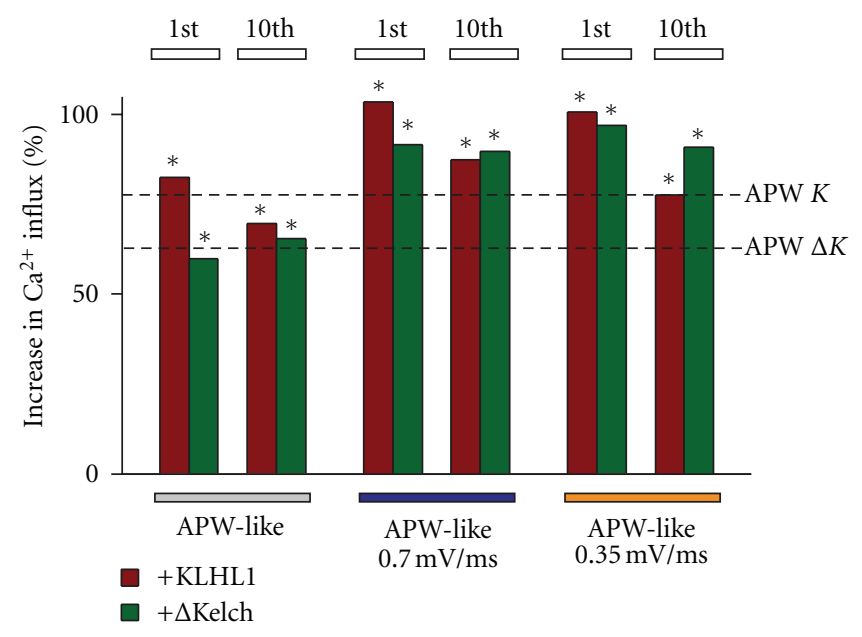

(b)

Figure 7: The APW-like stimulus produces an effect similar to an actual APW. (a) Stimulus (APW-like) created to replicate an actual APW (top) and APW-like stimuli with AHP slopes of $0.70 \mathrm{mV} / \mathrm{ms}$ and $0.35 \mathrm{mV} / \mathrm{ms}$ were compared. (b) Percentage of increase in calcium influx obtained with the stimuli shown above with respect to control; the magnitude of the changes induced by the original APWs is shown with dotted lines $(K=\mathrm{KLHL} 1 ; \Delta K=\Delta$ Kelch $) ;{ }^{*} P<0.05$.

$(n=15)$, whereas decreasing the hyperpolarization time resulted in an increase of $31 \%(n=22)$. In the case of KLHL1, all stimuli elicited significant increases in calcium influx compared to control (red bars). However, a decrease in the peak depolarization time resulted in a decrease of the effect of KLHL1 on $\alpha_{1 \mathrm{H}}$ as seen in Figure 3(b) (76\% versus $57 \%, n=19,16$, resp.). In contrast, a decrease in the hyperpolarization time to $6 \mathrm{~ms}$ resulted in calcium influx increase of $83 \%(n=6)$. Incidentally, the latter stimulus was ideal to eliminate current inactivation after the 10th train (Figure 3(b), red bars), possibly providing more time for recovery from inactivation. Still, none of these changes enabled a calcium influx increase in the presence of $\Delta$ Kelch thus, we next tested whether modification of the slope of repolarization has an effect on the calcium influx by $\Delta$ Kelch.

\subsection{SQAPs with Different Repolarization Rates Fail to Enable} Upregulation of $\mathrm{Ca}_{v} 3.2$ by $\Delta$ Kelch. Two new stimuli with different repolarization rates were tested, an SQAP with a repolarization slope of $70 \mathrm{mV} / \mathrm{ms}$ in contrast to the instantaneous upstroke in the SQAP $(120 \mathrm{mV} / \mathrm{ms})$ (blue and black traces, resp., Figure 4(a)) and a waveform with a slower repolarization rate of $35 \mathrm{mV} / \mathrm{ms}$. Overall, slowdown of the rate of repolarization did not rescue the effect of $\Delta$ Kelch, as seen from the values represented in Figure 4(b). For the first stimulus, $\Delta$ Kelch induced an increase in calcium influx of $25 \%(n=38)$, as reported previously, whereas the altered waveforms elicited increases of 45 and 33\%, respectively $(n=$ $20,19)$. The alteration of the slope rate resulted in calcium influxes of 78 and $65 \%$ above control for these stimuli in the presence of KLHL1 $(n=14,14)$, compared to $76 \%$ with SQAP $(n=19)$; all these values are significantly higher than control. Incidentally, the calcium influx by $\mathrm{Ca}_{\mathrm{v}} 3.2$ after the 10th spike in the presence of KLHL1 appeared to be less inactivated when repolarization occurred with a slope as opposed to an instantaneous change in voltage (as in SQAP) (Figure 4(b)).

Given that neither the duration of depolarization, the duration of the hyperpolarization step, nor the slope of repolarization significantly altered the calcium influx by $\Delta$ Kelch, we next targeted the AHP slope step in the action potential waveform.

3.5. The AHP Step in the APW Is a Major Determinant Allowing the Interaction between $\mathrm{Ca}_{v} 3.2$ and $\triangle$ Kelch and Thus Enabling Increased Calcium Influx by the Truncation Mutant. To determine whether the afterhyperpolarization slope step is important in the APW to enable the calcium influx increase seen with $\triangle$ Kelch, we modified the SQAP protocol to include afterhyperpolarization ramps of varying slopes. These protocol waveforms are depicted in Figure 5(a), which included an afterhyperpolarizing slope of $0.75 \mathrm{mV} / \mathrm{ms}$, a faster slope of $20 \mathrm{mV} / \mathrm{ms}$, and slower slope of $0.38 \mathrm{mV} / \mathrm{ms}$. As seen in the data shown in panel (b), changing the afterhyperpolarization step from a square pulse (seen in Figures 3 and 4) to a slope (Figure 5) enables the interaction between $\alpha_{1 \mathrm{H}}$-KLHL1. The protocol with an faster slope $(20 \mathrm{mV} / \mathrm{ms})$ enabled the least calcium influx, although its effect was still significant (57 and $52 \%$ for KLHL1 and $\Delta$ Kelch $)(n=12,12)$. On the other hand, the slower slope protocol $(0.38 \mathrm{mV} / \mathrm{ms})$ induced much higher calcium influx than the protocol with a slope of $0.75 \mathrm{mV} / \mathrm{ms}$ (69 and $68 \%$ increase for KLHL1 and $\Delta$ Kelch, resp. $),(n=12,14)$, comparable to the effect of the protocol with $0.75 \mathrm{mV} / \mathrm{ms}$ slope, clearly demonstrating that slower after-hyperpolarizing slopes also enable increased calcium influx through $\mathrm{Ca}_{\mathrm{v}} 3.2$ and more significantly allow the interaction between $\alpha_{1 \mathrm{H}}$ and $\Delta$ Kelch. 
We next combined the effects of the introduction of a repolarizing slope of $70 \mathrm{mV} / \mathrm{ms}, 35 \mathrm{mV} / \mathrm{ms}$, or instantaneous repolarization, combined with an afterhyperpolarization slope of $0.75 \mathrm{mV} / \mathrm{ms}$ in all traces, as depicted in Figure 6(a). The latter AHP slope value was selected due to its similarity to the actual slope of the action potential $(\sim 0.78 \mathrm{mV} / \mathrm{ms})$.

As seen in panel (b), all protocols elicited increased influx comparable with the control APW in the presence of KLHL1 (dotted lines, 76\% increase); for the WT protein (KLHL1), influx was 77, 89, and $77 \%$ of control $(n=22$, $18,18)$. Similarly, $\Delta$ Kelch elicited increases of 69,95 , and $74 \%$ in calcium influx $(n=24,19,20)$. Clearly, the afterhyperpolarization slope is paramount in enabling the interaction between the KLHL1 and $\alpha_{1 H}$; introduction of a repolarizing slope induced the maximum increase in calcium influx observed thus far for $\Delta$ Kelch.

Finally, we generated three protocols similar in shape to the original APW recorded from hippocampal neurons (Figure 7(a)). An APW-like protocol was generated with a repolarizing slope of $62 \mathrm{mV} / \mathrm{ms}$ (compared to $66 \mathrm{mV} / \mathrm{ms}$ of the original APW) and an afterhyperpolarization slope of $0.75 \mathrm{mV} / \mathrm{ms}$ (compared to $0.78 \mathrm{mV} / \mathrm{ms}$ ). We produced another two waveforms with slight variations from the original: an APW-like protocol with a repolarizing slope from hyperpolarization of $0.70 \mathrm{mV} / \mathrm{ms}$ and a second waveform with a slower slope of $0.35 \mathrm{mV} / \mathrm{ms}$. The results from these experiments are shown in Figure 7(b), which shows an enhancement of calcium influx concomitant with the slowdown of the afterhyperpolarization slope. For KLHL1, calcium influx was 83,104 , and $101 \%(n=9,19,19)$, and similarly, $\Delta$ Kelch enhanced Ca influx by 60,92 , and $97 \%$ $(n=12,22,22)$, corroborating the original APW data.

The experiments in this section clearly establish the importance of the AHP phase of the action potential for $\alpha_{1 \mathrm{H}}$ calcium influx. Moreover, this step allows the interaction between the mutant KLHL1 lacking the actin-binding Kelch motifs with the channel; this suggests that the deactivation step of $\alpha_{1 \mathrm{H}}$ channels is crucial to determine the interaction of the channel with the mutant ABP.

\section{Discussion}

The Kelch domain in KLHL1 is vital in the modulation of $\mathrm{Ca}_{\mathrm{v}} 3.2$ channels by KLHL1. In the absence of the $\beta$ propeller ( $\Delta$ Kelch), KLHL1 can still colocalize and interact with $\alpha_{1 \mathrm{H}}$ and elicit changes in $\mathrm{Ca}_{\mathrm{v}} 3.2$ calcium influx, albeit only with action potential stimuli [1]. Thus, KLHL1 requires its actin-binding Kelch domain to exert its effect on $\mathrm{Ca}_{\mathrm{v}} 3.2$. However, elimination of this domain uncovered another weaker interaction site between the channel and KLHL1. This interaction could only be observed during stimulation with action potentials. We investigated the properties within the action potential waveform that enable the interaction between $\alpha_{1 \mathrm{H}}$ and KLHL1.

Action potential waveform stimuli have been used to identify the specific contribution of calcium and other ionic currents during the physiological stimulus [11-13] and to determine how the currents' biophysical properties influence this contribution [14-16], see [17]. The shape and duration of the action potential are essential in determining the magnitude of neuronal calcium influx, specially given the importance of $\mathrm{Ca}_{\mathrm{v}} 3.2$ function in physiological processes such as adrenaline release from chromaffin cells, GABA release from thalamic reticular neurons, and in pain sensation [18-20]. Here, we modified the duration of the depolarization and the afterhyperpolarization steps, the repolarization slope, and the AHP slope. We found that a decrease of the plateau of depolarization tended to reduce $\mathrm{Ca}$ influx and that an increase in the length of hyperpolarization eliminated the frequency-dependent inactivation at $10 \mathrm{~Hz}$, although neither of these changes rescued the effect of $\Delta$ Kelch. Alteration of the slope of the repolarization phase did not rescue the effect of $\Delta$ Kelch, although the slower rates of repolarization appeared to increase the calcium influx at the last spike of the stimulus [13].

In contrast, we established that the slope of AHP is the crucial step that enables the interaction between $\alpha_{1 \mathrm{H}}$ and $\Delta$ Kelch. That this step resulted in an increase in calcium influx by $\mathrm{Ca}_{\mathrm{v}} 3.2$ is not a surprise, given the importance of tail currents in general in calcium entry [17], and specifically for $\mathrm{Ca}_{\mathrm{v}} 3.2$ with its slow kinetics of tail current deactivation. Thus, the transition step from open to closed state is necessary in the conformational change required to enable the interaction of regions in KLHL1 - other than the Kelch domains-with the $\alpha_{1 \mathrm{H}}$ subunit. In summary, KLHL1 interacts with $\alpha_{1 \mathrm{H}}$ with at least two different regions of the protein; the main interaction involves the Kelch domains and requires stabilization by the actin cytoskeleton. This dominant interaction is responsible for the effects of KLHL1 on the channels, such as the increase in current density by increased endosomal recycling and channel number at the membrane, and the changes in kinetic in the time constant of deactivation. In the absence of this primary interaction, a secondary, more labile interaction is detected. This interaction is responsible for increases in calcium influx only seen when the protein is subjected to an AP stimulus; specifically, the afterhyperpolarization slope of the $\mathrm{AP}$ appears to be paramount in enabling this interaction, which suggests that the transition from open to closed state of the channel is necessary and allows a conformation change in $\alpha_{1 \mathrm{H}}$ conducive to its interaction with $\Delta$ Kelch.

\section{Acknowledgments}

This paper is based upon work supported by the National Science Foundation under Grants nos. 0641141 and 1022075 (E. S. Piedras-Rentería). This research was also supported by a Stritch School of Medicine Dean's Research Award, Loyola University Chicago (E. S. Piedras-Rentería).

\section{References}

[1] K. A. Aromolaran, K. A. Benzow, L. L. Cribbs, M. D. Koob, and E. S. Piedras-Rentería, "T-type current modulation by the actin-binding protein Kelch-like 1," American Journal of Physiology, vol. 298, no. 6, pp. C1353-C1362, 2010.

[2] J. P. Nemes, K. A. Benzow, and M. D. Koob, "The SCA8 transcript is an antisense RNA to a brain-specific transcript 
encoding a novel actin-binding protein (KLHL1)," Human Molecular Genetics, vol. 9, no. 10, pp. 1543-1551, 2000.

[3] M. D. Koob, M. L. Moseley, L. J. Schut et al., "An untranslated CTG expansion causes a novel form of spinocerebellar ataxia (SCA8)," Nature Genetics, vol. 21, no. 4, pp. 379-384, 1999.

[4] Y. He, T. Zu, K. A. Benzow, H. T. Orr, H. B. Clark, and M. D. Koob, "Targeted deletion of a single Sca8 ataxia locus allele in mice causes abnormal gait, progressive loss of motor coordination, and Purkinje cell dendritic deficits," The Journal of Neuroscience, vol. 26, no. 39, pp. 9975-9982, 2006.

[5] K. A. Aromolaran, K. A. Benzow, L. L. Cribbs, M. D. Koob, and E. S. Piedras-Rentería, "Kelch-like 1 protein upregulates Ttype currents by an actin-F dependent increase in $\alpha(1 \mathrm{H})$ channels via the recycling endosome," Channels, vol. 3, no. 6, pp. 402-412, 2009.

[6] K. A. Aromolaran, K. A. Benzow, M. D. Koob, and E. S. Piedras-Rentería, "The Kelch-like protein 1 modulates P/Qtype calcium current density," Neuroscience, vol. 145, no. 3, pp. 841-850, 2007.

[7] R. Mortensen, J. D. Chesnut, J. P. Hoeffler et al., "Introduction of DNA into mammalian cells," in Short Protocols in Molecular Biology, F. M. Ausubel, R. Brent, R. E. Kingston et al., Eds., pp. 9.1-9.24, John Wiley \& Sons, New York, NY, USA, 1999.

[8] R. C. Lambert, Y. Maulet, J. L. Dupont et al., "Polyethylenimine-mediated DNA transfection of peripheral and central neurons in primary culture: probing $\mathrm{ca}^{2+}$ channel structure and function with antisense oligonucleotides," Molecular and Cellular Neuroscience, vol. 7, no. 3, pp. 239-246, 1996.

[9] J. Chemin, A. Monteil, E. Perez-Reyes, E. Bourinet, J. Nargeot, and P. Lory, "Specific contribution of human T-type calcium channel isotypes ( $\alpha 1 \mathrm{G}, \alpha 1 \mathrm{H}$ and $\alpha 1 \mathrm{l})$ to neuronal excitability," Journal of Physiology, vol. 540, no. 1, pp. 3-14, 2002.

[10] A. S. Kozlov, F. McKenna, J. H. Lee et al., "Distinct kinetics of cloned T-type $\mathrm{Ca}^{2+}$ channels lead to differential $\mathrm{Ca}^{2+}$ entry and frequency-dependence during mock action potentials," European Journal of Neuroscience, vol. 11, no. 12, pp. 41494158, 1999.

[11] T. Doerr, R. Denger, and W. Trautwein, "Calcium currents in single SA nodal cells of the rabbit heart studied with action potential clamp," Pflugers Archiv European Journal of Physiology, vol. 413, no. 6, pp. 599-603, 1989.

[12] D. P. McCobb and K. G. Beam, "Action potential waveform voltage-clamp commands reveal striking differences in calcium entry via low and high voltage-activated calcium channels," Neuron, vol. 7, no. 1, pp. 119-127, 1991.

[13] R. S. Scroggs and A. P. Fox, "Multiple $\mathrm{Ca}^{2+}$ currents elicited by action potential waveforms in acutely isolated adult rat dorsal root ganglion neurons," The Journal of Neuroscience, vol. 12, no. 5, pp. 1789-1801, 1992.

[14] I. M. Raman and B. P. Bean, "Resurgent sodium current and action potential formation in dissociated cerebellar Purkinje neurons," The Journal of Neuroscience, vol. 17, no. 12, pp. 4517-4526, 1997.

[15] D. B. Wheeler, A. Randall, and R. W. Tsien, "Changes in action potential duration alter reliance of excitatory synaptic transmission on multiple types of $\mathrm{Ca}^{2+}$ channels in rat hippocampus," The Journal of Neuroscience, vol. 16, no. 7, pp. 22262237, 1996.

[16] A. D. Randall and R. W. Tsien, "Contrasting biophysical and pharmacological properties of T-type and R-type calcium channels," Neuropharmacology, vol. 36, no. 7, pp. 879-893, 1997.
[17] B. P. Bean, "The action potential in mammalian central neurons," Nature Reviews Neuroscience, vol. 8, no. 6, pp. 451-465, 2007.

[18] V. Bisagno, M. Raineri, V. Peskin et al., "Effects of T-type calcium channel blockers on cocaine-induced hyperlocomotion and thalamocortical GABAergic abnormalities in mice," Psychopharmacology, vol. 212, no. 2, pp. 205-214, 2010.

[19] V. Jevtovic-Todorovic and S. M. Todorovic, "The role of peripheral T-type calcium channels in pain transmission," Cell Calcium, vol. 40, no. 2, pp. 197-203, 2006.

[20] V. Carabelli, A. Marcantoni, V. Comunanza et al., "Chronic hypoxia up-regulates $\alpha 1 \mathrm{H}$ T-type channels and low-threshold catecholamine secretion in rat chromaffin cells," Journal of Physiology, vol. 584, no. 1, pp. 149-165, 2007. 

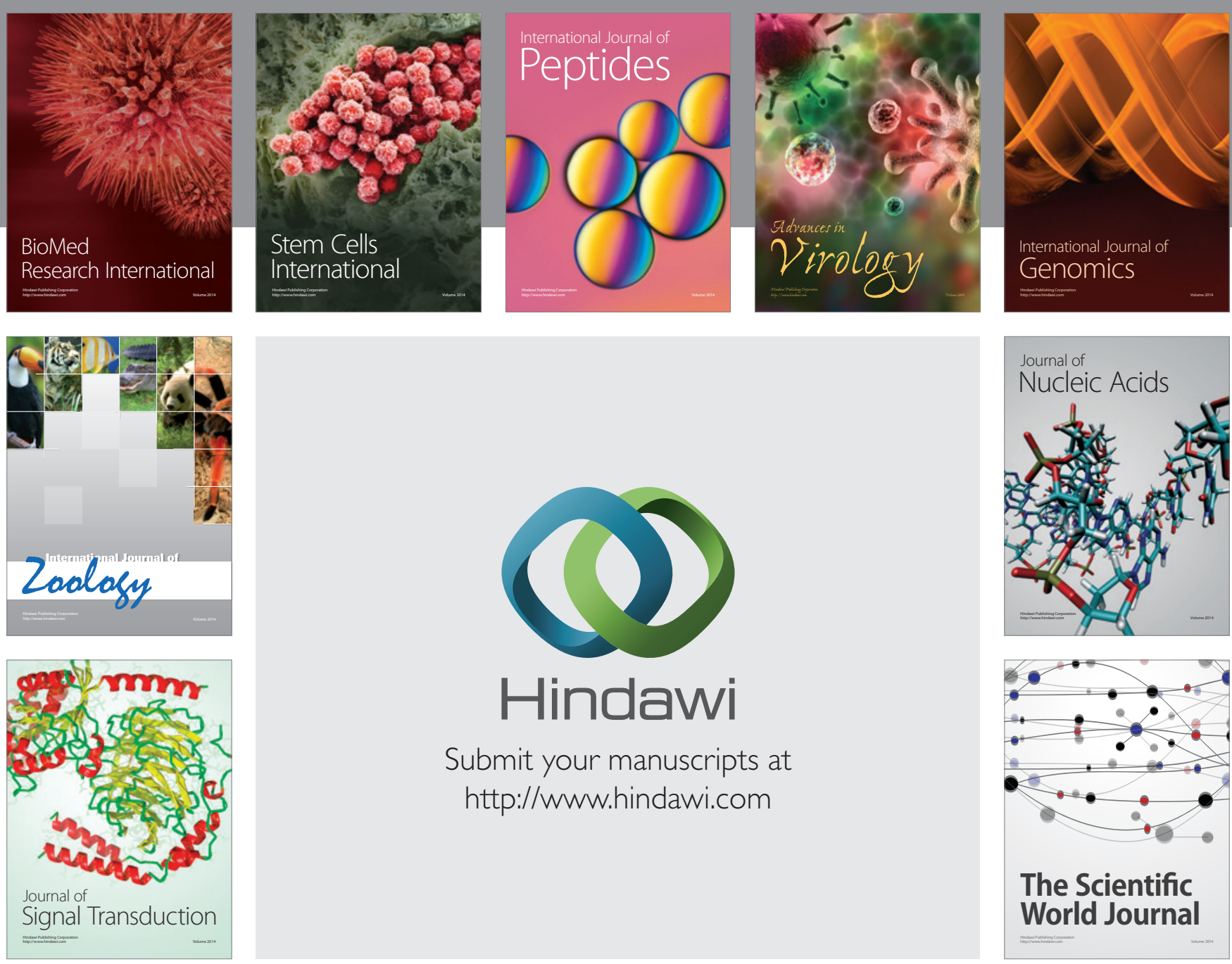

Submit your manuscripts at

http://www.hindawi.com
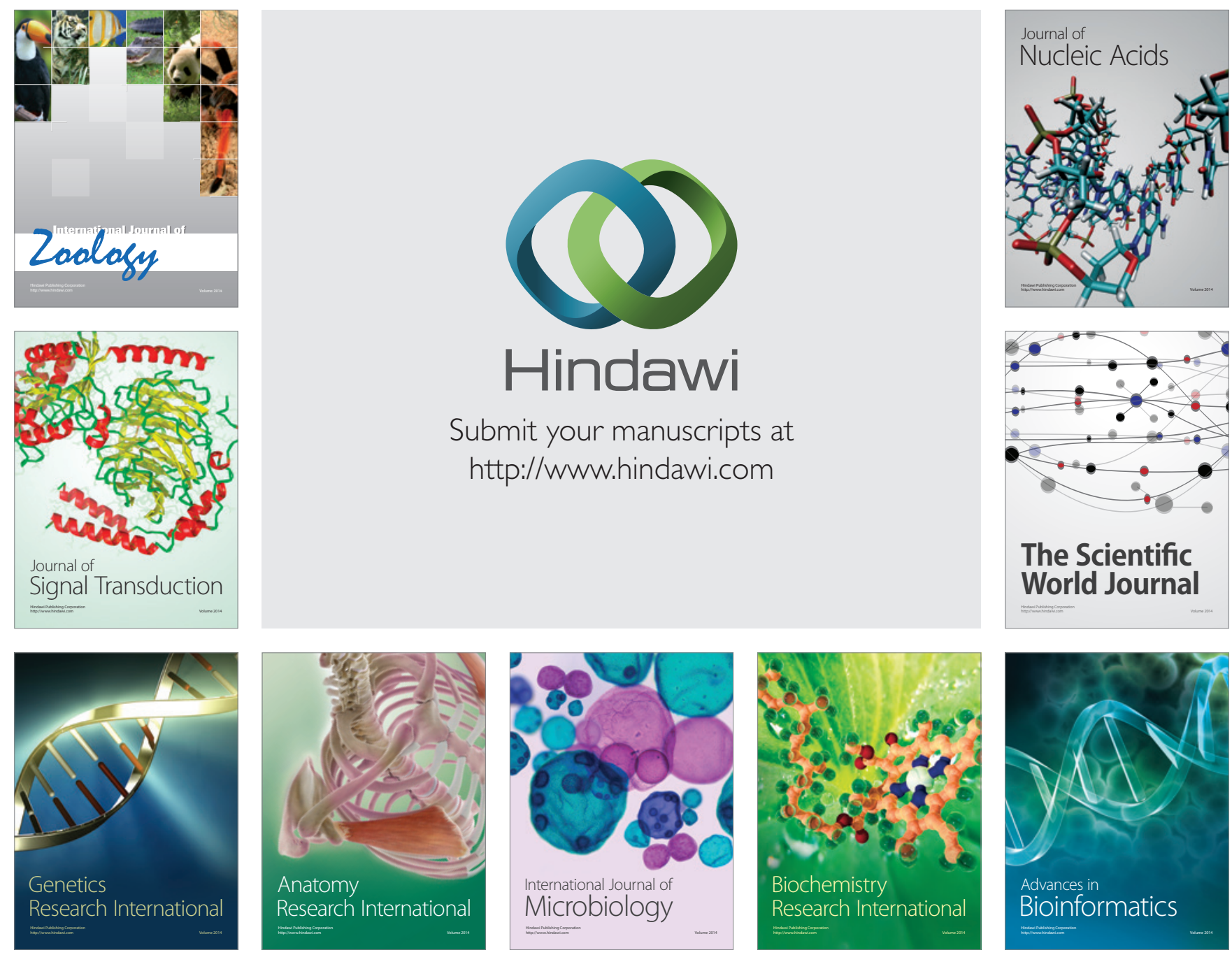

The Scientific World Journal
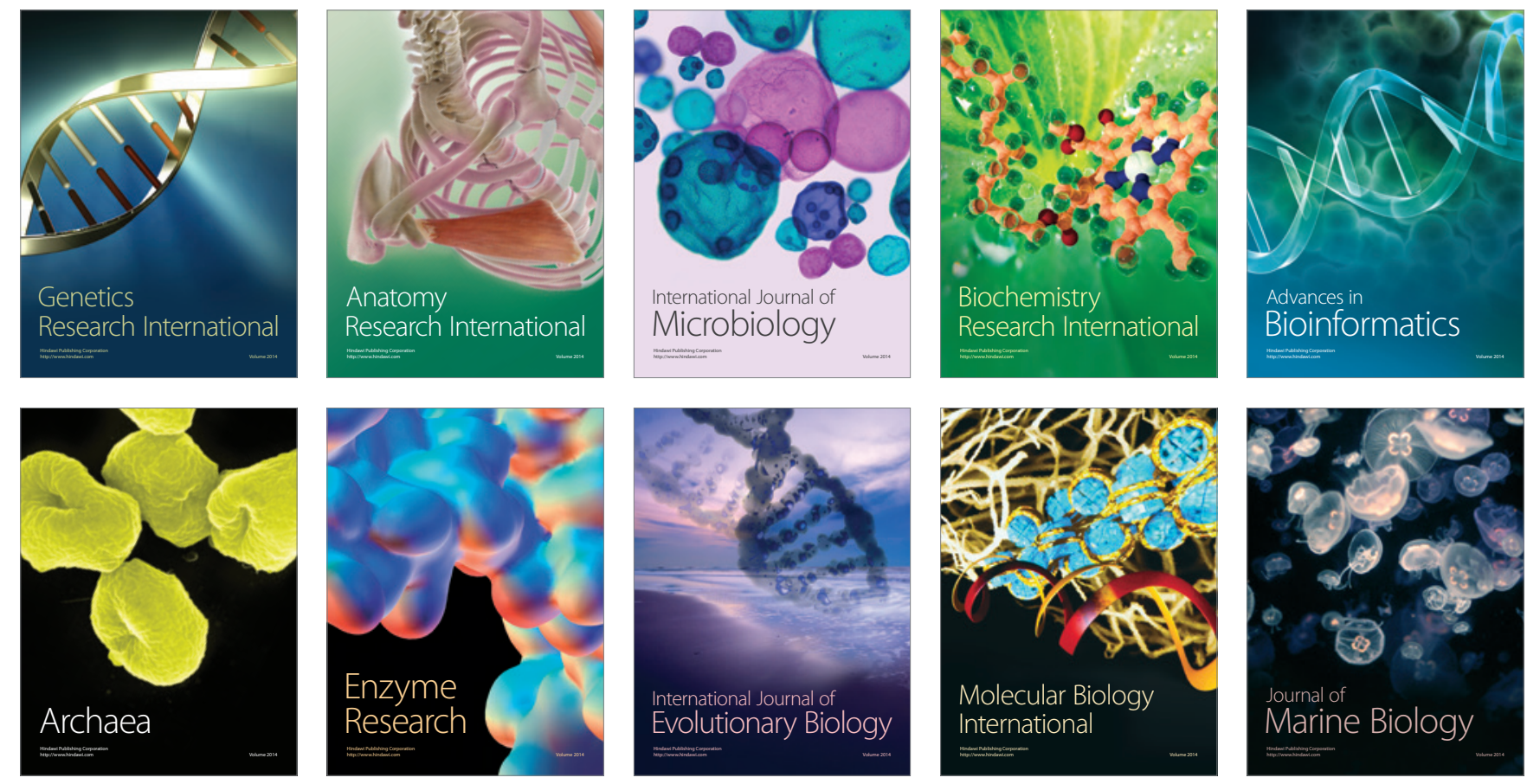